\title{
Analysis of optical systems by wave vectors
}

Ichirou Yamaguchi

Ichirou Yamaguchi, "Analysis of optical systems by wave vectors," Proc. SPIE 3190, Fifth International Topical Meeting on Education and Training in Optics, (8 December 1997); doi: 10.1117/12.294370

Event: Fifth International Topical Meeting on Education and Training in Optics, 1997, Delft, Netherlands 


\title{
Analysis of optical systems by wave vectors
}

\author{
Ichirou Yamaguchi \\ Optical Engineering Laboratory, \\ The Institute of Physical and Chemical Research(RIKEN) \\ Wako, Saitama, 351-01 Japan.
}

\begin{abstract}
Plane wave expansion of optical fields is well known in optical textbooks. The wave vector is normal to the wavefront and has the magnitude indicating the angular spatial frequency of the fields. Maxwell equations lead to the equations connecting the wave vector and temporal angular frequency, so-called, dispersion relations. The relations are derived for isotropic dielectric, metal, and anisotropic crystals. Then laws of reflection and refraction are derived from continuity of the wave vector components along the boundary. Geometrical construction based on the condition is shown for refraction into isotropic materials and crystals. Evanescent waves arising from total reflection are also formulated from the construction. Then formation of interference fringes between two plane waves propagating in different directions is graphically displayed and optical beat signals generated between different frequencies are explained in terms of the movement of the fringe patterns. Finally diffraction by periodic structures is constructed together with the difference between thin and thick gratings is also discussed. The case of a moving grating is also discussed.
\end{abstract}

Keywords: plane wave expansion, Maxwell equations, reflection, refraction, interference, diffraction

\section{INTRODUCTION}

Optical education comprises many disciplines such as geometrical optics, lens design, physical optics based on electromagnetic theory, quantum optics including nonlinear optics and lasers, electronic and mechanical design for optical components and electronics, interaction of light with matters needed for development of new materials and devices. One of the most important requirements for education is to deliver a small number of basic viewpoints or models from which many relationships and rules can be deduced. There are many good, complete, but thick textbooks for the above disciplines, but such simple basic concepts tying various optical phenomena have not yet been found by the author. One of them seems to be the concept of wave vectors that represents plane waves, the simplest but general tools for analysis of optical systems because arbitrary optical fields can be represented by their superposition.

In this paper I will show several examples of description of optical phenomena such as freespace propagation, reflection and refraction, interference, and diffraction by using the concepts of wave vectors. Mathematical relationships will also be illustrated by graphical constructions of wave vectors.

\section{WAVE VECTORS AND MAXWELL EQUATIONS}

\subsection{Plane waves and Maxwell equations}

Plane wave expansion of optical fields into angular spectrum is a basis for the Fourier optics that is very simple and useful for evaluation of optical systems ${ }^{1}$. A plane wave is represented by

$$
\boldsymbol{F}(\boldsymbol{r}, t)=\boldsymbol{A} \exp [i(\boldsymbol{k} \cdot \boldsymbol{r}-\omega t+\phi)],
$$

where $\boldsymbol{A}$ is the amplitude and $\boldsymbol{k}$ is the wave vector whose direction is normal to the wavefront and whose magnitude is given by $k=2 \pi / \lambda$ with the wavelength $\lambda$. The wave vector has three dimensions corresponding to the position vector $r$. Since arbitrary optical fields can be represented by superposition of the plane waves having different wave vectors and angular frequencies, it is enough to study the behaviors of elementary plane wave. 
First we consider the Maxwell equations that are fundamental for wave optics. They consist of four basic equations and three material equations. The first group of equations are given by
1) Ampere-Maxwell's law: $\quad \operatorname{rot} \boldsymbol{H}=\frac{\partial \boldsymbol{D}}{\partial t}+\boldsymbol{j}$
2) Faraday's law: $\quad \operatorname{rot} E=-\frac{\partial B}{\partial t}$

3) Coulomb-Gauss's law: $\operatorname{div} D=\rho$

4) No magnetic monopole: $\operatorname{div} B=0$

The material equations that result from macroscopic averaging are expressed as

1) Electric polarization: $\quad \boldsymbol{D}=\boldsymbol{\varepsilon} \boldsymbol{E}$

2) Ohm's law: $\quad j=\sigma E$

3) Magnetic polarization: $\boldsymbol{B}=\mu \boldsymbol{H}$

All of these three equations assume linear responses in electric and magnetic polarization as well as electric current .

If we substitute Eq.(1) into Eqs.(2a-d) with $\rho=0$, we obtain

$$
\begin{aligned}
& \boldsymbol{k} \times \boldsymbol{H}=i \omega \boldsymbol{D}+\sigma \boldsymbol{E}, \\
& \boldsymbol{k} \times \boldsymbol{E}=-i \omega \boldsymbol{B} \\
& \boldsymbol{k} \cdot \boldsymbol{D}=0 \\
& \boldsymbol{k} \cdot \boldsymbol{B}=0
\end{aligned}
$$

The last two equations indicate that the wave vector is normal to $\boldsymbol{D}$ and $\boldsymbol{B}$, while the first two ones lead to orthogonality between $\boldsymbol{H}$ and $\boldsymbol{D}$ and that between $\boldsymbol{E}$ and $\boldsymbol{B}$. Therefore, it follows that $\boldsymbol{D}, \boldsymbol{E}$, and $\boldsymbol{k}$ are coplanar as shown in Fig.1. It also holds in crystals where $\boldsymbol{D}$ and $\boldsymbol{E}$ are not parallel to each other.
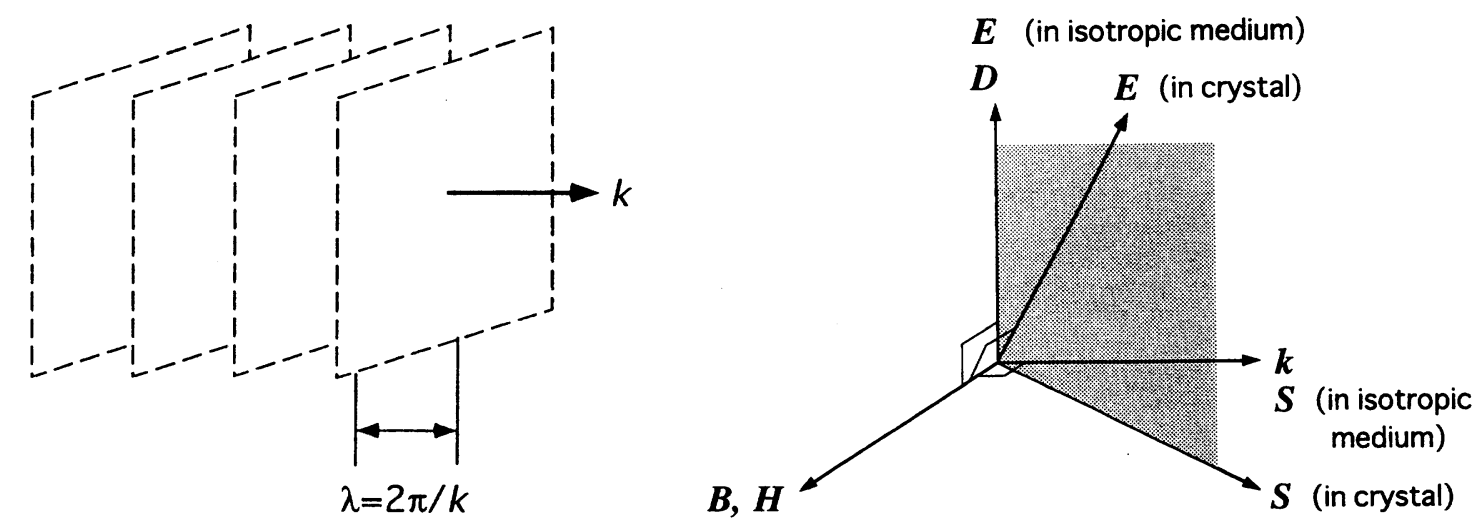

Fig.1 Relationship between wave vector and electromagnetic fields. 


\subsection{Dispersion relations}

In the case of isotropic transparent media we can set $\sigma=0$ in Eq.(4a) and obtain after elimination of $\boldsymbol{H}$ from Eqs. $(4 a, b)$ considering Eqs. $(3 a, c)$ the relation

$$
\left(|\boldsymbol{k}|^{2}-\varepsilon \mu \omega^{2}\right) \boldsymbol{E}=0
$$

that leads to the wave number independent of the electric field $\boldsymbol{E}$

$$
k \equiv|k|=\sqrt{\varepsilon \mu} \omega=\frac{n \omega}{c}=n k_{0},
$$

where $n$ is the refractive index and $c$ and $k_{0}$ mean the light velocity and the wave number in vacuum. We see that the magnitude of the wave vector is proportional to the refractive index. In vacuum the wave vector is proportional to the frequency but in dispersive media with $n(\omega)$ it shows nonlinear dependence on the frequency.

For absorbing media the conductivity $\sigma$ is finite and the wave vector satisfies the relation

$$
\left(|\boldsymbol{k}|^{2}-\varepsilon \mu \omega^{2}-i \mu \sigma \omega\right) \boldsymbol{E}=0
$$

The wave number becomes complex such as

$$
k=\alpha+i \beta=\omega \sqrt{\mu\left(\varepsilon+i \frac{\sigma}{\omega}\right)}=\omega \sqrt{\mu \tilde{\varepsilon}}
$$

with the complex dielectric constant $\tilde{\varepsilon}$. The plane wave decays after propagation by a distance characterized by $1 / \beta$. The complex refractive index is expressed as

$$
\tilde{n}=\frac{c k}{\omega}=n^{\prime}+i n^{\prime \prime}
$$

with the real part

$$
n^{\prime}=\frac{\alpha c}{\omega}=\sqrt{\frac{\varepsilon \mu}{2 \varepsilon_{0} \mu_{0}}\left[\sqrt{1+\left(\frac{\sigma}{\varepsilon \omega}\right)^{2}}+1\right]}
$$

and the imaginary part

$$
n^{\prime \prime}=\frac{\beta c}{\omega}=\sqrt{\frac{\varepsilon \mu}{2 \varepsilon_{0} \mu_{0}}\left[\sqrt{1+\left(\frac{\sigma}{\varepsilon \omega}\right)^{2}}-1\right]}
$$

In crystals where the electric displacement $\boldsymbol{D}$ is not in general parallel to the electric field such as

$$
\boldsymbol{D}=[\varepsilon] \boldsymbol{E}
$$

with the dielectric tensor $[\varepsilon]$ the dispersion relation contains the electric field such as

$$
\boldsymbol{k}(\boldsymbol{k} \cdot \boldsymbol{E})-|\boldsymbol{k}|^{2} \boldsymbol{E}-\mu \omega^{2}[\varepsilon] \boldsymbol{E}=0 .
$$


Hence the magnitude of the wave vector depends on its direction and is also connected with polarization of the electric field $\boldsymbol{E}$. The dependence of the magnitude on its direction is expressed by the index surfaces. Propagation of plane waves in a uniaxial crystal is illustrated in Fig.2. In the crystal they consist of a sphere and an ellipsoid. They cross each other at a single optic axis along which plane waves of any polarization state of $\boldsymbol{E}$ propagates at the same velocity. The wave corresponding to the sphere is called ordinary wave and behaves like plane wave in isotropic media, namely, its wave vector is normal to $\boldsymbol{D}$ and $\boldsymbol{E}$ which are mutually parallel and normal to the plane formed by the optic axis and $\boldsymbol{k}$ , so-called, the principal plane. On the other hand, the wave corresponding to the ellipsoid, called extraordinary wave, has the electric field $\boldsymbol{E}$ that is inclined to $\boldsymbol{D}$. Hence the direction of the Poynting vector $\boldsymbol{S}=\boldsymbol{E} \times \boldsymbol{H}$ does not coincide with $\boldsymbol{k}$. This direction is also called the ray direction connected with energy flow whose velocity is expressed ${ }^{2}$ by

$$
v_{r j}=\frac{\partial \omega}{\partial k_{j}} \quad(j=x, y, z) .
$$

This expression also gives the normal on the index surface and is analog to that of group velocity in dispersive media which is given by

$$
v_{g}=\frac{\partial \omega}{\partial k}
$$

A difference is that in crystals the frequency $\omega$ depends not only on the magnitude but also on the direction of the wave vector. If we consider dispersion of crystal in addition, the direction of the optic axis also depends on frequency. In biaxial crystals that have two optical axes these axes do not coincide with the ray axes along which the ray velocities associated with each of the index surfaces are identical.
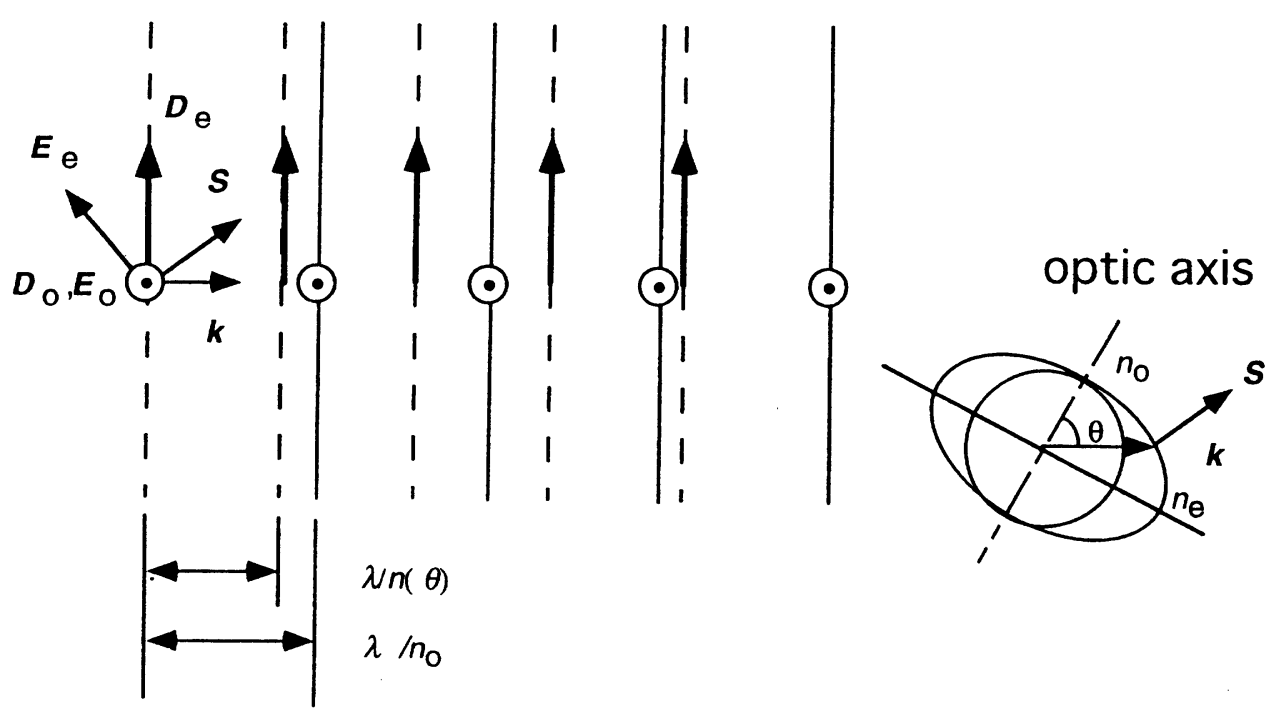

Fig.2 Wave vectors and the index surface in a uniaxial crystal.

\section{REFLECTION AND REFRACTION}

\subsection{Laws of reflection and refraction}


We can derive the laws of reflection and refraction from continuity of the tangential components of the wave vectors. Physically it means the connection of wavefronts in both media at the boundary as shown in Fig.3. Whereas the temporal frequency should be the same in the both media, the spatial frequency of the waves are required to be congruent at the boundary.

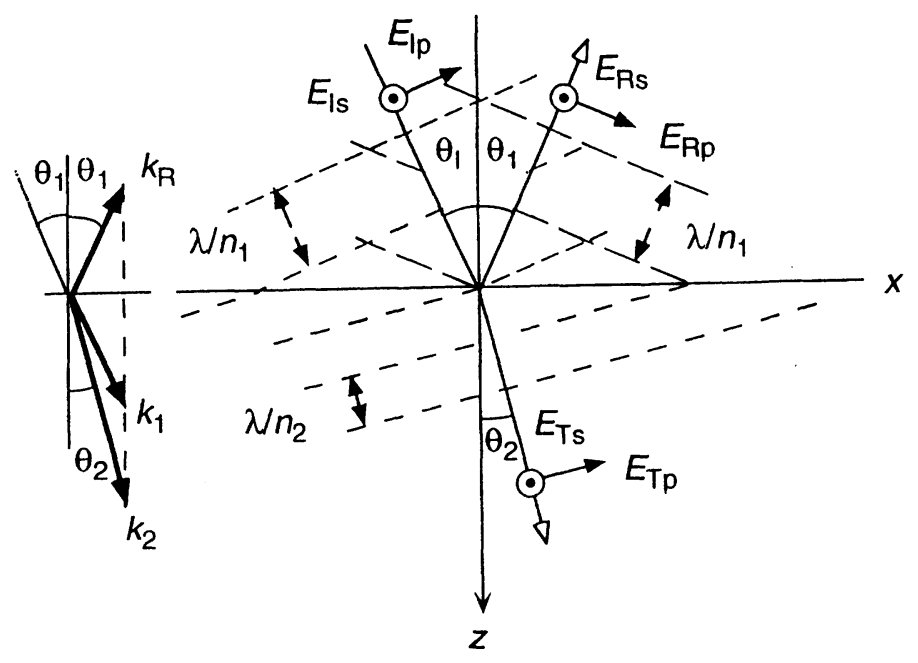

Fig.3 Continuity of the wave vector components and its physical meaning.

Constructions of reflection and refraction in isotropic media are illustrated in Fig.4(a). One circle has the radius of the refractive index $n_{1}$ in the first medium, while the solid semi-circle has $n_{2}$ in the second medium. At the Brewster angle satisfying $\tan \theta \mathrm{B}=n_{2} / n_{1}$ the incident direction goes through the crossing point of the normal tangentials at each of the circles.
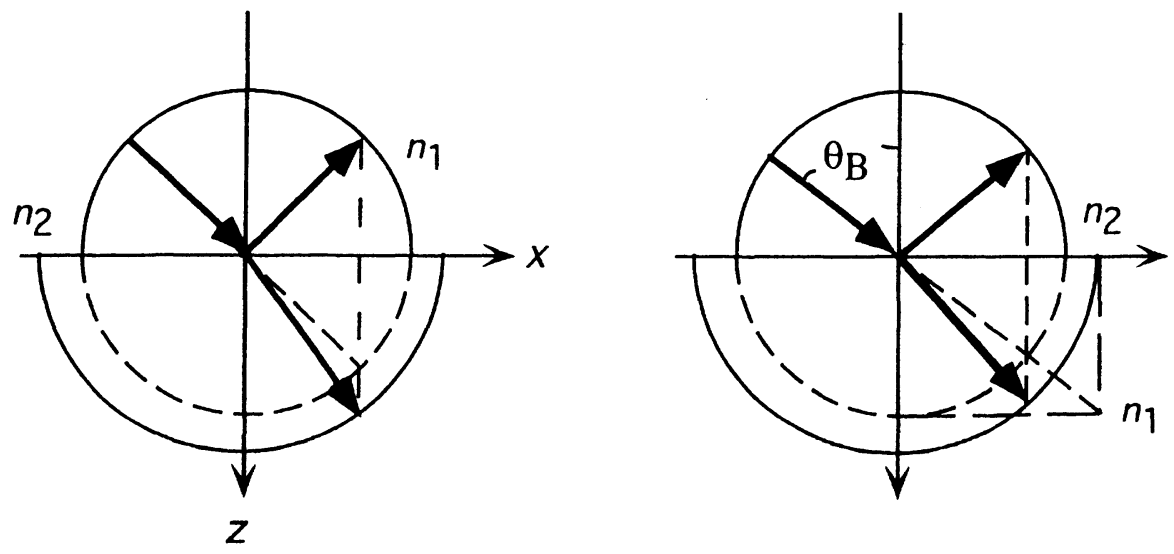

Brewster angle

Fig.4 Construction for the directions of refraction and reflection between isotropic media.

Refraction of wave vectors at a crystal surface can be constructed by using the cross-section of the index surfaces. In Fig. 5 we present examples with various directions of optic axis of a uniaxial crystal. The ray direction is given by the normal at the index surfaces. 3,4 

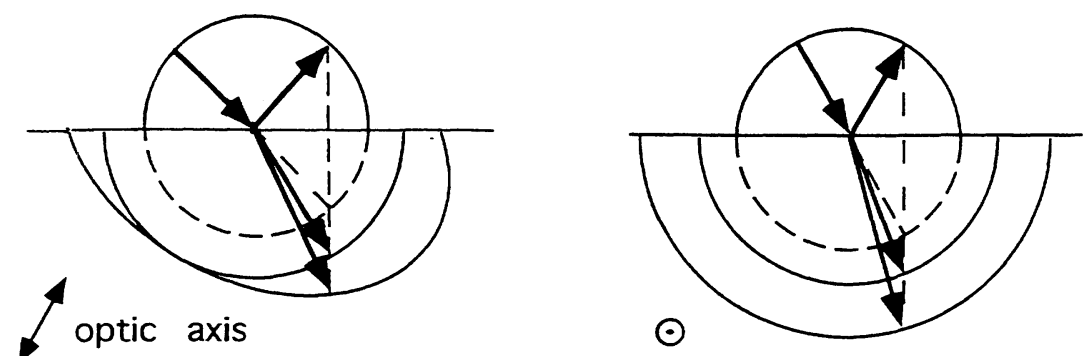

optic axis

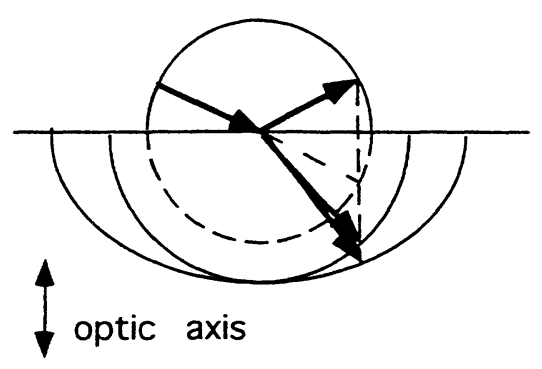

Fig.5 Wave vectors at reflection and refraction at a uniaxial crystal.

\subsection{Fresnel's coefficients}

The Fresnel's coefficients for reflection and transmission at the boundary can also be expressed in terms of components or projections of wave vectors along the surface normal. The reflection coefficient of p-components can be written as

$$
\rho_{p}=\frac{n_{1} \cos \theta_{2}-n_{2} \cos \theta_{1}}{n_{1} \cos \theta_{2}+n_{2} \cos \theta_{1}}=\frac{k_{1} / \cos \theta_{1}-k_{2} / \cos \theta_{2}}{k_{1} / \cos \theta_{1}+k_{2} / \cos \theta_{2}}
$$

in terms of the projection along the normal as shown in Fig.6a, while the s-component has the coefficient given by

$$
\rho_{s}=\frac{n_{1} \cos \theta_{1}-n_{2} \cos \theta_{2}}{n_{1} \cos \theta_{1}+n_{2} \cos \theta_{2}}=\frac{k_{1 z}-k_{2 z}}{k_{1 z}+k_{2 z}}
$$

that depends on the normal components of the wave vectors as shown in Fig.6b. The transmission coefficients are similarly represented as

$$
\tau_{p}=\frac{2 n_{1} \cos \theta_{1}}{n_{1} \cos \theta_{2}+n_{2} \cos \theta_{1}}=\frac{k_{1} / \cos \theta_{2}}{k_{1} / \cos \theta_{1}+k_{2} / \cos \theta_{2}}
$$

and

$$
\tau_{s}=\frac{2 n_{1} \cos \theta_{1}}{n_{1} \cos \theta_{1}+n_{2} \cos \theta_{2}}=\frac{k_{1 z}}{k_{1 z}+k_{2 z}} .
$$

Only in the case of transmission of p-polarization the cross-term between the both media appears in the numerator. 


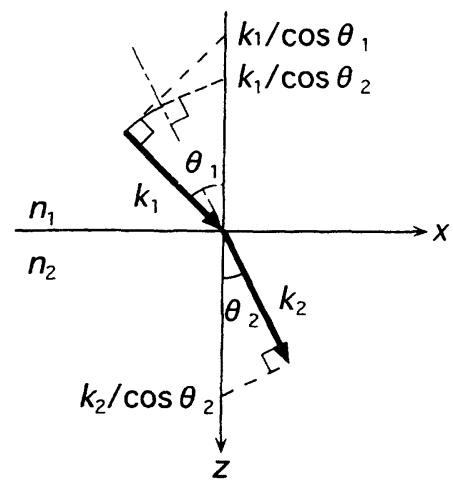

(a)

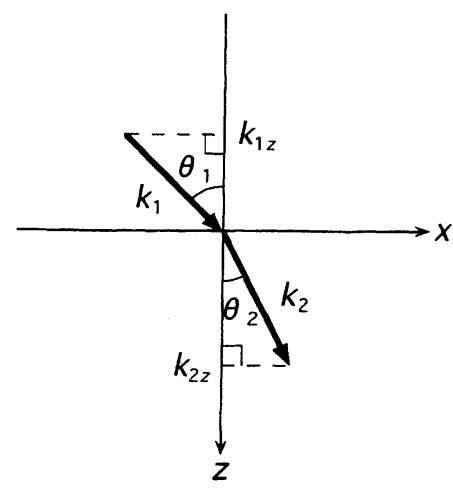

(b)

Fig.6 Components of wave vectors contributing to reflection and transmission coefficients.

\subsection{Total reflection}

When the second medium has lower refractive index than the first one, the construction becomes as shown in Fig.7. At the critical angle that satisfies $\sin \theta_{c}=n_{2} / n_{1}$ and can be constructed as shown in Fig. 7b, reflectivity becomes unity. For larger incident angle the wave vector in the second medium has the $\mathrm{z}$-component that is represented by the imaginary number

$$
k_{2 z}=\frac{2 \pi i \sqrt{n_{1}^{2} \sin ^{2} \theta_{1}-n_{2}^{2}}}{\lambda}=i k_{2 z}^{\prime}
$$

The value of $k_{2 z}{ }^{\prime}$ can be obtained from the crossing point of the surface normal with the hyperbola which is the branch of the circle for the incident angle larger than the critical angle. In this case tangential component $k_{2 \mathrm{x}}=k_{1 \mathrm{x}}$ exceeds the magnitude of $k_{2}$. The wave with the imaginary component of the wave vector is evanescent as in absorbing media mentioned before. The penetration depth below the boundary is represented by $1 / k_{2 z}{ }^{\prime}$ that decreases as the incident angle increases. Since the tangential component of the wave vector in the second medium is always real, the evanescent wave progresses along the boundary. It is called inhomogeneous wave because the planes of equal amplitude and those of equal phase do not coincide with each other.

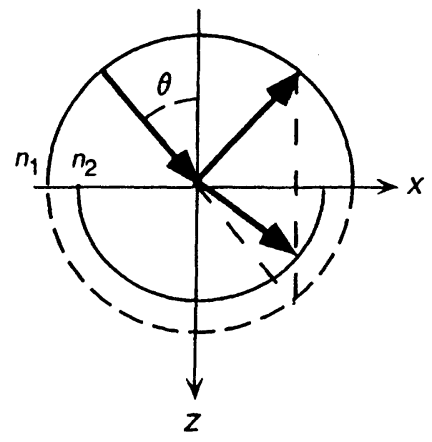

(a) $\theta<\theta_{\mathrm{C}}$

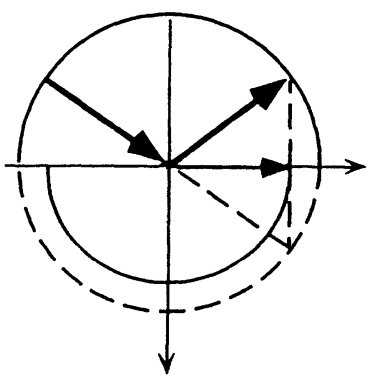

(b) $\theta=\theta_{\mathrm{C}}$

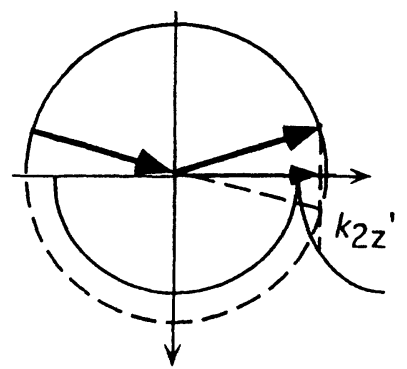

(c) $\theta>\theta_{\mathrm{C}}$

Fig.7 Wave vectors in incidence on an optically thin medium. 


\section{INTERFERENCE}

Interference between two plane waves with the wave vector $k_{1}$ and $k_{2}$ produces the fringe pattern expressed by

$$
\begin{aligned}
I(\boldsymbol{r}) & =\left|\boldsymbol{A}_{1} \exp \left[i\left(\boldsymbol{k}_{1} \cdot \boldsymbol{r}-\omega t+\phi_{1}\right)\right]+\boldsymbol{A}_{2} \exp \left[i\left(\boldsymbol{k}_{2} \cdot \boldsymbol{r}-\omega t+\phi_{2}\right)\right]\right|^{2} \\
& =\left|\boldsymbol{A}_{1}\right|^{2}+\left|\boldsymbol{A}_{2}\right|^{2}+2 \boldsymbol{A}_{1} \cdot \boldsymbol{A}_{2} \cos \left[\left(\boldsymbol{k}_{1}-\boldsymbol{k}_{2}\right) \cdot \boldsymbol{r}+\boldsymbol{\phi}_{1}-\phi_{2}\right]
\end{aligned}
$$

The interference term has the argument that depends on the position in the same manner as the plane wave with respect to the difference between the wave vectors represented by

$$
K=k_{1}-k_{2} .
$$

This vector can be named the fringe vector because its projection onto a plane of observation determines both the normal and the angular spatial frequency of the observed fringes. When the angle $\theta$ between the wave vectors is smaller than $45^{\circ}$, the cross section of the fringe patterns can be illustrated in terms of the moire fringes that are formed by the superposition of two linear gratings representing also the crosssections of the plane waves as shown in Fig.8(a) The magnitude of the fringe vector is given by

$$
|\boldsymbol{K}|=\sqrt{2|\boldsymbol{k}|^{2}-2 \boldsymbol{k}_{1} \cdot \boldsymbol{k}_{2}}=k \sqrt{2(1-\cos \theta)}=k \sin \frac{\theta}{2} .
$$

Hence the spacing of the interference pattern is equal to

$$
\Lambda=2 \pi / K=\lambda / \sin \frac{\theta}{2}
$$

that takes the minimum value $\lambda / 2$ when the waves progress in the opposite direction as illustrated in Fig.8(b). In this case the wave resultant from superposition is a perfect standing wave.

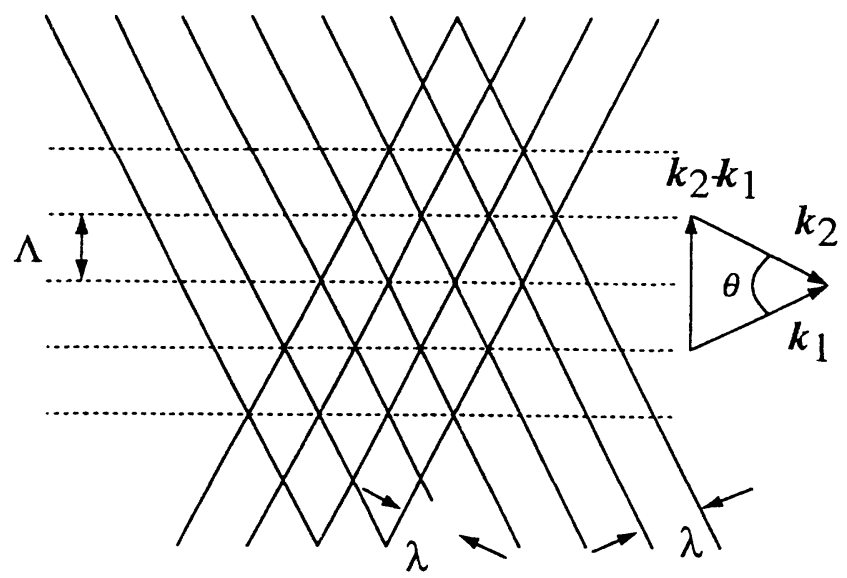

(a)

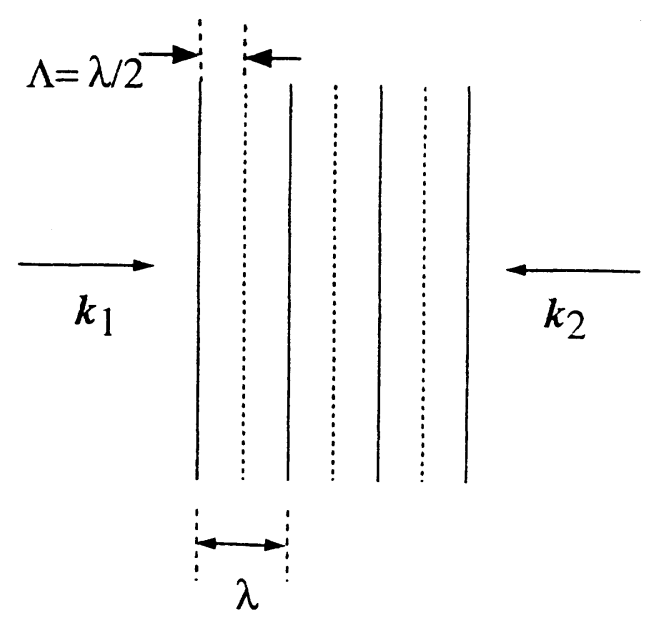

(b)

Fig.8 Illustration of interference between two plane waves. 
When two plane waves with different frequencies $\omega_{1}$ and $\omega_{2}$ are superposed, the resultant intensity becomes

$$
I(\boldsymbol{r}, t)=\left|\boldsymbol{A}_{1}\right|^{2}+\left|\boldsymbol{A}_{2}\right|^{2}+2 \boldsymbol{A}_{1} \cdot \boldsymbol{A}_{2} \cos \left[\left(\boldsymbol{k}_{1}-\boldsymbol{k}_{2}\right) \cdot \boldsymbol{r}-\left(\omega_{1}-\omega_{2}\right) t+\phi_{1}-\phi_{2}\right]
$$

The interference term shows now exactly the same form as the plane wave. The velocity of the moving fringe pattern normal to the fringes is given by

$$
V=\frac{\omega_{1}-\omega_{2}}{\left|\boldsymbol{k}_{1}-\boldsymbol{k}_{2}\right|}=\frac{\Omega}{K}
$$

with the difference of the angular frequency

$$
\Omega=\omega_{1}-\omega_{2}
$$

usually called the beat frequency. The fringe velocity of Eq.(23) takes the same expression as the group velocity given by Eq.(15). We have to notice that Eq. (22) is valid only if the beat is much lower than the optical frequencies. If we detect the interference intensity at a fixed point, a sinusoidal signal with the beat frequency, which preserves the phase difference between the waves. This is the principle of heterodyne interferometry. The fringe movement mentioned above can actually visualized as that of the moire fringes when one of the gratings is moved. This also constitutes a picture for the basic principle of the displacement measurement by moire methods or interferometry.

We can say that the interference pattern arises as the spatial beat signal between wave vector components on an observation plane. This model might be useful for analyzing formation of general patterns that are produced by superposition of many plane wave components with various amplitude and phase relationships.

\section{DIFFRACTION}

We consider diffraction of plane wave at a periodic structure whose grating vector is expressed by $K \mathrm{~g}$. The vector is directed normal to the grating grooves and has the angular spatial frequency of the grating. Then the wave vector of the $m$-th diffraction order can be expressed by

$$
\boldsymbol{k}_{m}=\boldsymbol{k}+\boldsymbol{m} \boldsymbol{K}_{g}
$$

This relation is derived from the condition for the constructive interference between the plane waves diffracted at the neighboring grooves. Since the wavelength should be unchanged in this case, the construction for the diffracted orders can be illustrated as in Fig. 9(a). When the x-component of $\boldsymbol{k}_{\boldsymbol{m}}$ exceeds the magnitude of the incident wave vector, its normal component $k_{m z}$ becomes complex and is determined from the intersection at the hyperbola similar to the situation in total reflection. The amplitude of the diffracted orders depends on the shape of grooves.

When the thickness of the grating is larger than the spacing, we also have to consider the coupling of the normal component of the vectors as well as the tangential components. Hence diffraction only occurs if Eq.(25) with $m=1$ is realized as shown in Fig.9(b).

Diffraction from a grating moving at the velocity $\boldsymbol{V}$ such as progressive acoustic wave gives rise to frequency shift given by

$$
\omega_{m}-\omega=m \boldsymbol{V} \cdot \boldsymbol{K}_{g}
$$

that corresponds to a Doppler shift. The frequency shift can be detected from the interference signal between different diffracted orders. The relationship of Eq.(26) corresponds to conservation of energy. 

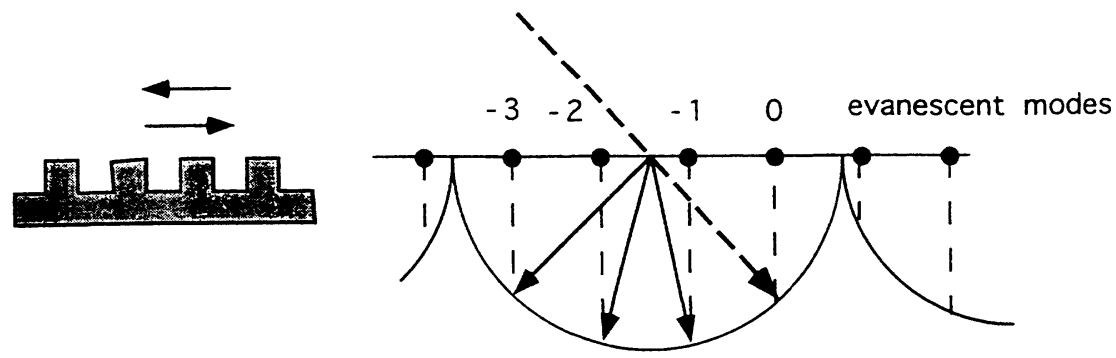

(a) Thin grating.
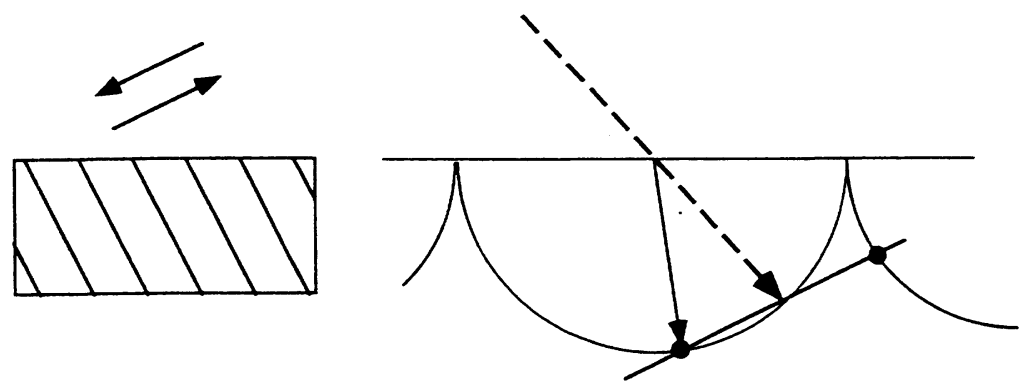

Bragg diffraction

(b) Volume grating.

Fig.9 Construction of diffraction orders from a thin grating and volume grating.

\section{CONCLUSIONS}

In this paper we have tried to describe various phenomena observed in wave optics by graphical construction using the wave vectors of plane wave component of optical fields. First we discussed plane wave solutions of Maxwell equations and the resulting dispersion relations for isotropic media and crystals. Especially propagation of ordinary and extraordinary waves in a uniaxial crystal was graphically illustrated. Next direction changes of wave vectors in reflection and refraction between different media were explained by using diagrams with index surfaces in the both media including crystals. Total reflection and the properties of the associated evanescent waves were also illustrated. Then combination of plane waves was introduced to visualize two-beam interference. Interpretation in terms of moire fringes was presented including those for heterodyne interferometry. Finally diffraction of a plane wave at periodic gratings was schematically depicted with reference to generation of evanescent waves, Bragg diffraction at volume grating, and diffraction at a moving grating.

Most of these descriptions can be found in various textbooks of optics separately. The unique contribution of this paper, however, might be to present them from a unified viewpoints and to show their physical meanings illustrated with geometrical constructions. For education and training visible images and demonstrations would be especially helpful for fixation and applications of acquired knowledge and experiences.

\section{REFERENCES}

1. For example, J. W. Goodmann, Introduction to Fourier Optics, McGraw-Hill, 1968.

2. L. D. Landau and E. M. Lifshitz, Electrodynamics of Continuous Media, Pergamon Press, 1965.

3. M. V. Klein, Optics, John-Wiley \& Sons, 1970.

4. A. Yariv and P. Yeh, Optical Waves in Crystals, John-Wiley \& Sons, 1984. 